\title{
Análise multicriterial no auxílio de tomada de decisão: Estudo de caso no reservatório São Gonçalo, Paraíba
}

\section{Multicriterial analysis in the decision-making aid: Case study in the São Gonçalo reservoir, Paraíba, Brazil}

\author{
Valterlin da Silva Santos ${ }^{1 *}$, Aíla Katamara Queiroga Nóbrega ${ }^{2}$
}

\begin{abstract}
Resumo: O reservatório São Gonçalo, situado no município de Sousa, Paraíba, entrou em colapso em agosto de 2015, de modo que as populações das cidades abastecidas pelo reservatório sofreram com um racionamento de água e as atividades agrícolas no Perímetro Irrigado de São Gonçalo e nas áreas no entorno do reservatório foram suspensas. Diante disso, objetivou avaliar a melhor forma de operação dos recursos hídricos do reservatório para que tal fato não ocorra novamente. Para tanto, foi proposto 5 (cinco) alternativas de operação do reservatório considerando os principais usos e os níveis de água do mesmo. Foi utilizado o método de análise multicriterial Prometheee II para a busca da melhor alternativa. Foram estabelecidos critérios de avaliação separados em duas dimensões (critérios referentes à utilização da água no reservatório e critérios referentes à utilização da água pela agricultura irrigada). A subjetividade da decisão humana foi analisada através de 11 (onze) cenários possíveis de pesos para cada dimensão. Os resultados apontaram que a alternativa 3 (suspensão da atividade de irrigação quando o nível o reservatório estiver abaixo de $20 \%$ da capacidade de armazenamento) e a alternativa 4 (diminuição da água destinada a irrigação quando o nível do reservatório atingir $40 \%$ da capacidade de armazenamento e suspensão da atividade de irrigação quando o nível o reservatório estiver abaixo de $20 \%$ da capacidade de armazenamento) são as melhores alternativas para a operação do reservatório dependendo do peso destinada a cada dimensão, porém tais alternativas também podem ser as piores.
\end{abstract}

Palavras-chave: Recursos hídricos; Processo decisório; Operação de reservatórios.

\begin{abstract}
The São Gonçalo reservoir, located in the municipality of Sousa, Paraíba, collapsed in August 2015, so that the populations of the cities supplied by the reservoir suffered from a rationing of water and the agricultural activity in São Gonçalo Irrigated Perimeter and in the areas around the reservoir were suspended. Therefore, the aim of this article is evaluate the best mode of operating the reservoir water resources so that this fact not occurs again. Thus, it was proposed five (5) alternatives of operating considering the main uses and water levels of reservoir. The multicriteria analysis method Prometheee II was used to find the best alternative. The were established evaluate criteria in two dimensions (criteria for the use of water in the reservoir and criteria for the use of water by irrigated agriculture). The subjectivity human decision was analyzed through 11 (eleven) possible weight scenarios for each dimension. The results indicated that alternative 3 (suspension of irrigation when the reservoir level is below $20 \%$ of the storage capacity) and alternative 4 (decrease of water intended for irrigation when the reservoir level reaches $40 \%$ of the storage capacity and suspension of the irrigation activity when the reservoir level is below $20 \%$ of the storage capacity) are the best alternatives for reservoir operation depending on the weight intended for each dimension, but this alternatives may also be the worst.
\end{abstract}

Key words: Water resources; Decision making; Reservoir operation.

\footnotetext{
Trabalho apresentado no IV Encontro Interdisciplinar da Paraíba realizado entre 09 a 10 de novembro de 2017 no Centro de Ciências Jurídicas e Sociais da Universidade Federal de Campina Grande, Sousa, Paraíba.

*Autor para correspondência

Recebido para publicação em 15/11/2017; aprovado em 27/11/2017

${ }^{1}$ Doutor em Recursos Naturais, Universidade Federal de Campina Grande, Sousa; (83) 99172-7913, valterlin@yahoo.com.br.

${ }^{2}$ Bacharel em Ciências Contábeis, Universidade Federal de Campina Grande, Sousa, aila_katamara@hotmail.com
} 


\section{INTRODUÇÃO}

A tomada de decisão é um desafio para qualquer situação que acarrete problemas e controvérsias de interesses. Por esse motivo é importante analisar várias alternativas, colher informações, dados, se valer de ferramentas que auxiliem a tomada de decisão diante do conhecimento prévio e escolher a alternativa que mais se adéqua aos objetivos a serem alcançados para que assim à decisão tomada seja mais segura (DUTRA; MOLIN, 2013).

A problemática da tomada de decisão nos dias atuais é caracterizada por um número crescente de alternativas e critérios conflitantes, dentre os quais os decisores necessitam selecionar, ordenar, classificar ou ainda descrever com detalhes as alternativas a serem selecionadas, considerando múltiplos critérios (ARAÚJO; ALMEIDA, 2009).

Com relação aos recursos hídricos, o processo de tomada de decisão envolve muitas variáveis e tem se desenvolvido cada vez mais para a resolução de conflitos, isso ocorre devido ao aumento da necessidade e da multiplicidade de usos. O processo de tomada de decisão está rodeado de incertezas, as quais irão influenciar os resultados obtidos de acordo com os processos de elaboração, formulação e modelação da tomada de decisão. E para minimizar as incertezas no processo de tomada de decisão existem técnicas e ferramentas que vem se desenvolvendo cada vez mais, auxiliando na eficiência das decisões (CHAVES, 2004).

Para a gestão e planejamento dos recursos hídricos são utilizadas várias ferramentas e métodos, com o intuito de dar suporte à tomada de decisão, direcionadas para a gestão continuada e ajustada ao uso coerente desses recursos. Tendo em vista que a utilização de recursos hídricos envolve interesses múltiplos e consequentemente conflitantes, vários são os atores e agentes que estão envolvidos e atuam no processo de decisão (POMPERMAYER et al., 2007).

A análise multicriterial é uma ferramenta de grande valia para auxiliar na tomada de decisão, tendo em vista a necessidade de avaliar uma alternativa através de vários critérios para assim tomar uma decisão mais segura. Essa análise busca elencar as alternativas mediante os fatores de relevância levantados anteriormente, para assim alcançar a melhor alternativa diante dos problemas encarados (CAMPOS, 2011).

Com essa finalidade existem muitos métodos de análise multicritério, tais métodos podem ser propostos de acordo com as situações identificadas auxiliando assim na escolha das alternativas de acordo com a definição e avaliação dos critérios (GOMES JÚNIOR et al., 2011).

A escolha do método a ser utilizada deve ser considerada a aplicabilidade do método ao problema em questão e levar em consideração as preferências dos decisores envolvidos, não deixando de lado também as vantagens e desvantagens desse método. Portanto para cada problema existirá um método mais apropriado que servirá de apoio a decisão mediante vários critérios elencados. Desta forma, a grande vantagem da análise multicritério é que independente da escolha do método, é que ela está ampara em uma modelagem robusta e transparente, apresentando premissas e preferências dos decisores relacionado aos objetivos considerados durante o processo decisório (FRANKLIN; FERNANDES, 2012).

Um método com bastantes aplicações da análise multicriterial é o método PROMETHEE II. Essa ferramenta agrupa par a par os indicadores observados e monta um ranking indicando da melhor à pior alternativa, desta forma sua utilização apóia consideravelmente a tomada de decisão mediante vários critérios (MOURA; COSTA, 2012).

Segundo Morais e Almeida (2006) este método foi proposto pela primeira vez em 1982 por Brans e Vincke, e desde então não deixou de ser objeto de desenvolvimento e adaptações complementares, tendo sido aplicado com sucesso em vários problemas de diferentes naturezas.

O método Promethee II apresenta vantagens importantes a serem consideradas, já que permite a avaliação de cenários diferentes inserindo diferentes critérios de interesses na análise, assim fornece muitas informações relevantes para auxiliar na tomada de decisão, além de obter uma aplicação de fácil entendimento (ALBUQUERQUE; NÚÑNEZ, 2010).

Aplicações do método PROMETHEE II, principalmente nas questões envolvendo o problema de recursos hídricos podem ser observados nos trabalhos de Carvalho e Curi, (2014), Morais et al.(2010), Zuffo et al. (2002), Carvalho et al. (2013), Carvalho et al. (2015).

Desde 2012, o Nordeste brasileiro está sofrendo as consequências da pior seca dos últimos 50 anos. $\mathrm{O}$ reservatório São Gonçalo, situado no município de Sousa PB entrou em colapso em agosto de 2015, as populações da zona rural e das cidades abastecidas pelo reservatório sofreram com um racionamento de água, sendo que desde janeiro de 2015 está proibida a retirada de água do reservatório para fins de irrigação de culturas agrícolas.

Diante dessa escassez hídrica enfrentada, Batista (2013) propôs 5 (cinco) alternativas para a melhor operação do reservatório. Os resultados obtidos mostraram que com a implantação de uma política de planejamento e controle do reservatório é possível garantir o atendimento sustentável das demandas de abastecimento humano. Com relação a demanda para a agricultura irrigada tem-se que as políticas de operações influenciam no grau de atendimento dessas demandas. Entretanto não foi possível estabelecer qual a melhor regra operacional para o reservatório

Assim este trabalho tem como objetivo avaliar qual a melhor alternativa de utilização dos recursos hídricos do reservatório São Gonçalo, com o auxilio do método multicriterial Promethee II.

\section{MATERIAL E MÉTODOS}

\section{Área de Estudo}

A área de estudo corresponde ao Reservatório São Gonçalo, localizado entre os municípios de Sousa, Marizópolis e Nazarezinho, na região do Alto Sertão do Estado da Paraíba (Figura 1). O reservatório pertence à Bacia do rio Piancó-Piranhas-Açu na Região do Alto Curso do Rio Piranhas.

As principais demandas hídricas supridas pelo reservatório São Gonçalo são: o abastecimento dos Municípios de Sousa (Distrito Sede, Distrito São Gonçalo e Núcleos Habitacionais I, II e III) e de Marizópolis; a demanda para abastecimento difuso de comunidades localizadas próximas ao reservatório; a irrigação do Perímetro Irrigado de São Gonçalo (PISG), localizado a jusante do reservatório; bem como a água retirada de forma pelos irrigantes que vivem ao redor do reservatório, conhecida como irrigação difusa, sendo essa não controlada pelos órgãos gestores do reservatório (BATISTA, 2013). 
Figura 1. Localização do Açude São Gonçalo na Região do Alto Curso do Rio Piranhas na região do Alto Sertão do Estado da Paraíba, Brasil

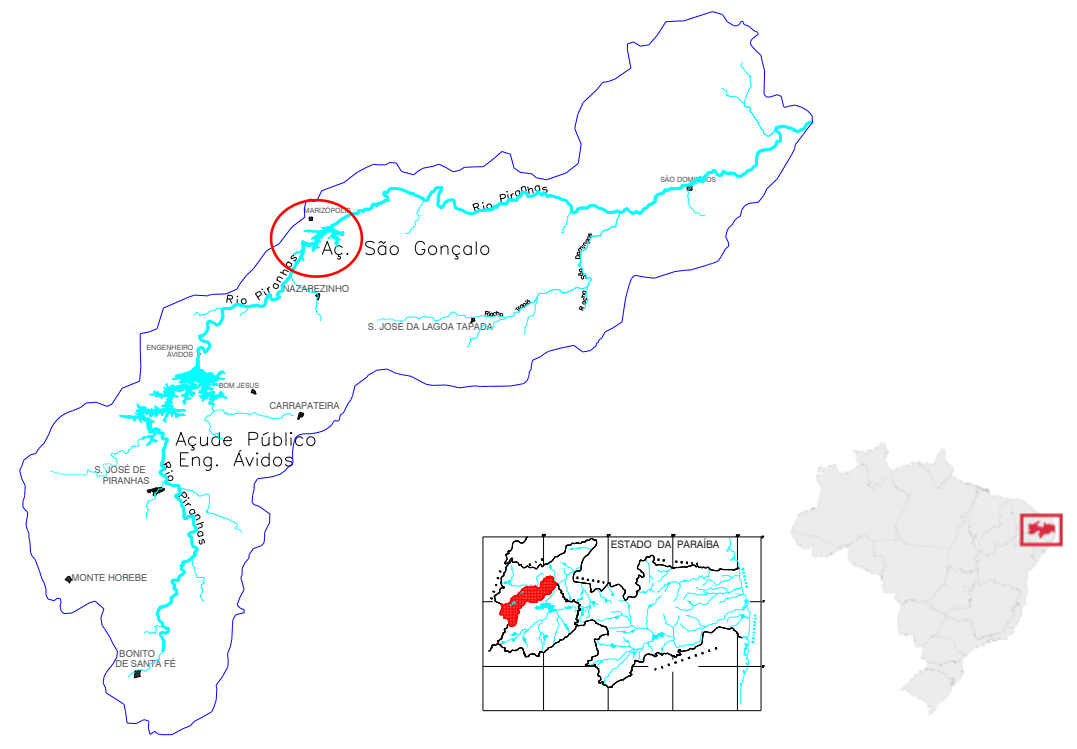

Fonte: Adaptado de SCIENTEC (1997).

\section{Proposta de Operação do Reservatório São Gonçalo}

Contudo, Batista (2013) propôs 5 alternativas para a operação do reservatório de São Gonçalo.

Alternativa1: Quando o volume reservatório encontrarse abaixo de $40 \%$ de sua capacidade máxima deverá ser suprimida parte da oferta destinada ao Perímetro Irrigado de São Gonçalo e à irrigação difusa das comunidades ribeirinhas, proporcionando-lhes apenas a chamada "irrigação de salvação", que equivale a $60 \%$ do volume normalmente liberado para essa finalidade.

Alternativa2: Quando o volume do açude encontrar-se abaixo de $30 \%$ de sua capacidade máxima deverá ser suprimida parte da oferta destinada ao Perímetro Irrigado de São Gonçalo e à irrigação difusa das comunidades ribeirinhas, proporcionando-lhes apenas a "irrigação de salvação".

Alternativa3: Quando o volume do reservatório ficar abaixo de $20 \%$ de sua capacidade máxima, deverá ocorrer à supressão completa da oferta de água destinada a irrigação, tanto a difusa quanto a do Perímetro Irrigado.

Alternativa4: Combinação das alternativas 1 e 3, ou seja, quando o reservatório estiver abaixo de $40 \%$ de sua capacidade máxima, deverá ser suprimida parte da oferta destinada ao Perímetro Irrigado de São Gonçalo e à irrigação difusa das comunidades ribeirinhas, proporcionando-lhes apenas a "irrigação de salvação"; e caso o volume fique inferior aos $20 \%$ da capacidade máxima, deve ocorrer a supressão completa da oferta liberada para a irrigação.

Alternativa5: Combinação das alternativas 2 e 3, ou seja, quando o reservatório estiver abaixo de $30 \%$ de sua capacidade máxima, deverá ser suprimida parte da oferta destinada ao Perímetro Irrigado de São Gonçalo e à irrigação difusa das comunidades ribeirinhas, proporcionando-lhes apenas a "irrigação de salvação"; e caso o volume fique inferior aos $20 \%$ da capacidade máxima, deve ocorrer a supressão completa da oferta liberada para a irrigação.

Constatou-se que em todas as alternativas, as demandas para abastecimento dos centros urbanos e abastecimento das comunidades ribeirinhas tiveram suas demandas plenamente atendidas. Entretanto as demandas Perímetro Irrigado de São Gonçalo e da irrigação difusa apresentaram falhas de atendimento de acordo com a alternativa escolhida.

\section{Critérios de Avaliação}

Deste modo, para auxiliar a tomada de decisão para escolha da melhor alternativa foram considerados critérios definidos a partir de duas dimensões do sistema: a Agricultura Irrigada e o Reservatório.

Os critérios para a Agricultura Irrigada têm o objetivo de retratar os anseios dos decisores quanto ao suprimento hídrico do PISG e da irrigação difusa em torno do reservatório. Assim foram estabelecidos os critérios de confiabilidade, resiliência ou elasticidade, vulnerabilidade e vazão média.

Confiabilidade: representa a probabilidade de atendimento da demanda, ou seja, é a percentagem de tempo em que a demandas foi atendida. A confiabilidade é entendia como sendo o oposto do risco e quanto mais próximo de 1 (um) for o seu valor, melhor será a confiabilidade do sistema.

Resiliência ou elasticidade: representa a probabilidade do sistema retorna de uma falha caso esta tenha ocorrido. É usado para avaliar o quão rapidamente o sistema retorna de um estado de falha para um estado satisfatório de atendimento da demanda. Algumas vezes é preferível um sistema que apresente muitas falhas, mas que se recupere mais rápido, do que um tenha poucas falhas e demore mais para se recuperar.

Vulnerabilidade: serve para mostrar o quão severa é a magnitude das falhas ao qual o sistema está sujeito, caso ela tenha ocorrido. Quanto maior o déficit hídrico, maior será a vulnerabilidade.

Vazão média: representa a quantidade de água fornecida por unidade de tempo ao PISG e a irrigação difusa. Quanto maior for seu valor significa que houve maior quantidade de água disponibilizada.

Os critérios para o reservatório têm o objetivo de retratar os anseios dos decisores quanto ao impacto da operação sobre o volume de água do reservatório. Assim foram estabelecidos os critérios de evaporação, vertimento, vazão efluente, volume $20 \%$.

Evaporação: representa a quantidade de água do reservatório transformada em vapor d'água devido à radiação solar e a temperatura do ar. Quanto maior for seu valor maior a será perda de água do reservatório.

Vertimento: representa a quantidade de água do reservatório que foi extravasada pelo vertedor. Quanto menor 
for seu valor maior foi à eficiência no uso da água reservatório.

Vazão efluente: representa a quantidade de água retirada do reservatório para o atendimento das demandas. Maiores valores podem impacta a disponibilidade de água do reservatório.

Volume 20\%: representa a probabilidade do volume de água do reservatório fica abaixo de $20 \%$ de sua capacidade de armazenamento. Quanto maior seu valor maior as chances do reservatório entra em colapso (seca completamente).

Segundo Santos (2009) as funções de preferência utilizadas no método Promethee II expressam a essência da preferência de uma alternativa sobre a outra com relação a cada critério. Procurou-se, quando possível, fazer uso de funções de preferência que pudessem incluir margens de erro ou tolerâncias tendo em vista que, ao estimar os valores para os critérios relativos às alternativas, eventuais erros poderão ser introduzidos nestes valores, desta maneira os parâmetros das funções possibilitarão a correção destes erros. Para isso, fez-se uso da função de preferência da função do tipo III.

A determinação dos pesos para os critérios de cada dimensão e o valor do parâmetro da função de preferência da função do tipo III podem ser observadas nos Quadros 1 e 2.

Quadro 1. Funções de preferência e pesos para os critérios da dimensão agricultura irrigada.

\begin{tabular}{|l|c|c|c|c|}
\hline \multicolumn{5}{|c|}{ Dimensão Agricultura Irrigada } \\
\hline \multirow{2}{*}{ Critério } & \multirow{2}{*}{ Objetivo } & Função de Preferência & \multirow{2}{*}{ Peso } \\
\cline { 3 - 4 } & & Tipo & Parâmetros & \\
\hline Confiabilidade & Max & III & $90 \%$ & $30 \%$ \\
\hline Resiliência & Max & III & $100 \%$ & $20 \%$ \\
\hline Vulnerabilidade & Min & III & $100 \%$ & $20 \%$ \\
\hline Vazão Média & Max & III & $1,27 \mathrm{~m}^{3} / \mathrm{s}$ & $30 \%$ \\
\hline \multicolumn{4}{|c|}{ Total } & $\mathbf{1 0 0 \%}$ \\
\hline
\end{tabular}

Quadro 2. Funções de preferência e pesos para os critérios da dimensão reservatório.

\begin{tabular}{|l|c|c|c|c|}
\hline \multicolumn{5}{|c|}{ Dimensão Reservatório } \\
\hline \multirow{2}{*}{ Critério } & \multirow{2}{*}{ Objetivo } & \multicolumn{2}{c|}{ Função de Preferência } & \multirow{2}{*}{ Peso } \\
\cline { 3 - 4 } & & Tipo & Parâmetros & \\
\hline Evaporação & Min & III & $1,1 \mathrm{hm}^{3}$ & $30 \%$ \\
\hline Vertimento & Min & III & $1,5 \mathrm{hm}^{3}$ & $20 \%$ \\
\hline Vazão afluente & Min & III & $1,25 \mathrm{~m}^{3} / \mathrm{s}$ & $20 \%$ \\
\hline Volume 20\%. & Min & III & $20 \%$ & $30 \%$ \\
\hline \multicolumn{4}{|r}{} & \multicolumn{4}{r|}{ Total } & $\mathbf{1 0 0 \%}$ \\
\hline
\end{tabular}

Em estudos que envolvem análise multicriterial, geralmente, é utilizada a técnica de aplicação de questionários com o objetivo de se poder representar nos pesos de cada dimensão os anseios dos participantes diretamente envolvidos com as ações a serem tomadas em cada alternativa que for escolhida. Entretanto o trabalho de campo seria enorme, incluindo dificuldades de acesso, e, muito provavelmente, ainda não seria representativo o suficiente. Além disso, a diferença cultural dos participantes envolvidos poderia levar a se formular questionários ou muito simples ou complexos, podendo até mesmo incorrer no erro da tendenciosidade dos aplicadores do questionário ao se tentar esclarecer as perguntas, que gerariam insatisfações (Santos et al., 2005).

Assim, no intuito de elimina uma possível tendenciosidade, oriunda da aplicação de questionários, e obter resultados mais consistentes, foi feito uma análise de sensibilidade, através de variações iguais a $10 \%$ no peso de cada dimensão (agricultura irrigada e reservatório) para o intervalo compreendido entre 0 e $100 \%$, de tal forma que a soma dos pesos atribuídos as dimensões resultasse em 100\%, totalizando 11 cenários possível como descrito na Tabela 1.

Tabela 1. Cenários e pesos atribuídos para cada dimensão.

\begin{tabular}{ccc} 
Cenários & Agricultura irrigada & Reservatório \\
\hline C1 & $0 \%$ & $100 \%$ \\
C2 & $10 \%$ & $90 \%$ \\
C3 & $20 \%$ & $80 \%$ \\
C4 & $30 \%$ & $70 \%$ \\
C5 & $40 \%$ & $60 \%$ \\
C6 & $50 \%$ & $50 \%$ \\
C7 & $60 \%$ & $40 \%$ \\
C8 & $70 \%$ & $30 \%$ \\
C9 & $80 \%$ & $20 \%$ \\
C10 & $90 \%$ & $10 \%$ \\
C11 & $100 \%$ & $0 \%$ \\
\hline
\end{tabular}

Contudo, a matriz de avaliação composta pelas 05(cinco) alternativas e os 08 (oito) critérios divididos nas 02 (duas) dimensões pode ser observada na Tabela 2.

\section{RESULTADOS E DISCUSSÃO}

Na Tabela 3 observa-se o resultado de todos os cenários para as duas dimensões (Agricultura Irrigada e Reservatório).

Nota-se que apenas as alternativas 3 e 4 configuram como a melhor alternativa para a operação do reservatório São Gonçalo.

Tabela 2. Matriz de avaliação.

\begin{tabular}{|c|c|c|c|c|c|c|c|}
\hline \multirow{2}{*}{ Dimensão } & \multirow{2}{*}{ Critério } & \multirow{2}{*}{ Unidades } & \multicolumn{5}{|c|}{ Alternativa } \\
\hline & & & 1 & 2 & 3 & 4 & 5 \\
\hline \multirow{4}{*}{ Agricultura irrigada } & Confiabilidade & $\%$ & 59 & 67 & 84 & 60 & 69 \\
\hline & Resiliência & $\%$ & 14 & 14 & 50 & 14 & 16 \\
\hline & Vulnerabilidade & $\%$ & 40 & 41 & 100 & 43 & 50 \\
\hline & Vazão Média & $\mathrm{m}^{3} / \mathrm{s}$ & 1,22 & 1,26 & 1,27 & 1,21 & 1,24 \\
\hline \multirow{4}{*}{ Reservatório } & Evaporação & $\mathrm{hm}^{3}$ & 1,03 & 0,98 & 0,96 & 1,05 & 1,00 \\
\hline & Vertimento & $\mathrm{hm}^{3}$ & 1,49 & 1,38 & 1,37 & 1,54 & 1,43 \\
\hline & Vazão efluente & $\mathrm{m}^{3} / \mathrm{s}$ & 1,38 & 1,42 & 1,43 & 1,37 & 1,41 \\
\hline & Volume $20 \%$. & $\%$ & 6 & 12 & 17 & 2 & 5 \\
\hline
\end{tabular}

Fonte: Adaptado de Batista (2013) 
Tabela 3. Avaliação das alternativas para todos os cenários para as proposta de operação do Reservatório São Gonçalo em Sousa, Paraíba

\begin{tabular}{|c|c|c|c|c|}
\hline \multirow{2}{*}{ Cenários } & \multicolumn{2}{|c|}{ Peso da dimensão } & \multirow{2}{*}{ Melhor Alternativa } & \multirow{2}{*}{ Pior Alternativa } \\
\hline & Agricultura irrigada & Reservatório & & \\
\hline 1 & $0 \%$ & $100 \%$ & Alternativa 4 & Alternativa 3 \\
\hline 2 & $10 \%$ & $90 \%$ & Alternativa 4 & Alternativa 3 \\
\hline 3 & $20 \%$ & $80 \%$ & Alternativa 4 & Alternativa 2 \\
\hline 4 & $30 \%$ & $70 \%$ & Alternativa 4 & Alternativa 2 \\
\hline 5 & $40 \%$ & $60 \%$ & Alternativa 3 & Alternativa 1 \\
\hline 6 & $50 \%$ & $50 \%$ & Alternativa 3 & Alternativa 1 \\
\hline 7 & $60 \%$ & $40 \%$ & Alternativa 3 & Alternativa 1 \\
\hline 8 & $70 \%$ & $30 \%$ & Alternativa 3 & Alternativa 1 \\
\hline 9 & $80 \%$ & $20 \%$ & Alternativa 3 & Alternativa 1 \\
\hline 10 & $90 \%$ & $10 \%$ & Alternativa 3 & Alternativa 4 \\
\hline 11 & $100 \%$ & $0 \%$ & Alternativa 3 & Alternativa 4 \\
\hline
\end{tabular}

Fonte: Elaboração própria.

A alternativa 3 (supressão completa da oferta de água destinada a irrigação quando o volume do reservatório ficar abaixo de $20 \%$ de sua capacidade máxima) passa a ser a melhor alternativa quando se considera um peso de pelo menos $40 \%$ para a dimensão Agricultura Irrigada (ou peso de até $60 \%$ para a dimensão Reservatório).

Essa alternativa de operação do reservatório resulta em uma confiabilidade de atendimento das demandas agrícolas em torno de $84 \%$ (maior entre todas as alternativas) e uma resilencia de $50 \%$ (a melhor entre todas as alternativas) com as menores perdas de água por evaporação e vertimento devido à maior retirada de água do reservatório (maior vazão efluente entre todas as alternativas).

Entretanto a mesma apresenta uma vulnerabilidade de $100 \%$ (maior entre todas as alternativas), ou seja, caso ocorra uma falha não haverá água para o atendimento das demandas agrícolas. Fato esse é preocupante, pois o tempo médio de retorno de uma falha é de 02 (dois) meses. Desse modo, espera-se, para essa alternativa de operação, que, caso ocorra uma falha, as culturas agrícolas do PISG e do entorno do reservatório ficarão, em média, 02 (dois) meses sem suprimento de água, o que pode comprometer o desenvolvimento da cultura agrícola e sua produtividade.

Além do mais, nessa alternativa, há maior chance (17\%) do reservatório apresenta um volume de água abaixo de $20 \%$ de sua capacidade de armazenamento, podendo o mesmo entrar em colapso (volume de água abaixo do volume morto).

Assim a alternativa 3 configura como à pior alternativa quando se considera um peso pelo menos $90 \%$ para a dimensão Reservatório (ou peso de até $10 \%$ para a dimensão Agricultura Irrigada).

A alternativa 4 (supressão parcial da oferta de água destinada a irrigação quando o volume do reservatório ficar abaixo de $40 \%$ de sua capacidade máxima e supressão completa quando o volume do reservatório ficar abaixo de $20 \%$ de sua capacidade máxima) passa a ser a melhor alternativa quando se considera um peso de pelo menos $70 \%$ para a dimensão Reservatório (ou peso de até $30 \%$ para a dimensão Agricultura Irrigada).

Essa alternativa apresenta a menor chance $(2 \%)$ do reservatório apresenta um volume de água abaixo de $20 \%$ de sua capacidade de armazenamento e a menor retirada de água do reservatório (menor vazão efluente entre todas as alternativas), porém apresenta as maiores perdas de água por evaporação e vertimento.
Tem-se nessa alternativa uma baixa confiabilidade de atendimento das demandas hídricas das culturas agrícolas do PISG e do entorno do reservatório, ou seja, espera-se que em $40 \%$ do tempo haverá alguma falha no atendimento dessas demandas, podendo essa falha se prolonga por mais de 07 (sete) meses. Porém, a magnitude da falha será em média de $43 \%$ do valor demanda, ou seja, mais de $60 \%$ do valor da demanda hídrica requerida pela cultura agrícola será fornecido.

Desse modo, a alternativa 4 configura como à pior alternativa quando se considera um peso pelo menos $90 \%$ para a dimensão Agricultura Irrigada (ou peso de até $10 \%$ para a dimensão Reservatório).

As alternativas 1 e 2 configuram como piores alternativas quando se estipula pesos entre $20 \%$ e $80 \%$.

A alternativa 5 não se apresenta como a melhor alternativa e nem como a pior alternativa em nenhum cenário podendo ser uma alternativa possível de operação de reservatório.

Sugere-se que a metodologia seja replicada em outras regiões com o intuito de auxiliar na operação de reservatórios. Outros critérios podem ser considerados, uma vez que a subjetividade na escolha dos critérios de análise e dos pesos cabe interpretações diferenciadas.

\section{CONCLUSÕES}

As melhores alternativas para a operação do reservatório São Gonçalo são a alternativa com supressão completa da oferta de água destinada a irrigação quando o volume do reservatório ficar abaixo de $20 \%$ de sua capacidade máxima quando se considera um peso de pelo menos $40 \%$ para a dimensão Agricultura Irrigada e a alternativa com supressão parcial da oferta de água destinada a irrigação quando o volume do reservatório ficar abaixo de $40 \%$ de sua capacidade máxima e supressão completa quando o volume do reservatório ficar abaixo de $20 \%$ de sua capacidade máxima caso contrario.

\section{REFERÊNCIAS}

ALBUQUERQUE, F. S.; NÚÑEZ, W. P. Critérios para a tomada de decisão em obras rodoviárias sustentáveis. Ambiente Construído, Porto Alegre, v. 10, n. 3, p.151-163, 2010. 
ARAÚJO, A. G.; ALMEIDA, A. T.. Apoio à decisão na seleção de investimentos em petróleo e gás: uma aplicação utilizando o método PROMETHEE. Gestão da Produção, São Carlos, v. 16, n. 4, p. 534-543, 2009.

BATISTA, R. L. Planejamento da utilização da água de um sistema hídrico: Um estudo de caso no reservatório São Gonçalo - Sousa/PB. 2013. 91 f. Trabalho de Conclusão de Curso (Graduação em Administração) - Universidade Federal de Campina Grande, Sousa. 2013.

CAMPOS, M. B. A. Métodos multicritérios que envolvem a tomada de decisão. 2011. 51 f. Monografia (Especialização em Matemática) - Universidade Federal de Minas Gerais, Belo Horizonte. 2011.

CARVAlHO, J. R. M. de; CURI, W. F. Sistema de Indicadores para a Gestão de Recursos Hídricos em Municípios: Uma Abordagem Através dos Métodos Multicritério e Mutidecisor. In: XVII SEMEAD Seminários em Administração, 2014, São Paulo. Anais ... São Paulo: FEA-USP, 2014.

CARVALHO, J. R. M. de; CURI, W. F; CURI, R. C. Uso da Análise Multicritério na Construção de Um Índice de Sustentabilidade Hidroambiental: Estudo em Municípios Paraibanos. Revista Brasileira de Gestão e Desenvolvimento Regional, Taubaté, v. 9, n. 2, p.3-26, 2013.

CARVAlHO, V. D. H. de; POLETO, T.; SILVA, L. C. e; COSTA, A. P. C. S. Abordagem Multicritério de Apoio a Decisões Estrategicamente Sustentáveis nas Organizações. Revista Produção Online, Florianópolis, v. 15, n. 3, p.925947, 2015.

CHAVES, E. M. B. Tomada de Decisão e Otimização de Alternativas no Planejamento com Múltiplos Objetivos em Unidades de Gerenciamento - Bacia do Rio dos Sinos (Rio Grande do Sul). 2004. 193 f. Tese (Doutorado em Recursos Hídricos e Saneamento Ambiental) - Universidade Federal do Rio Grande do Sul, Porto Alegre. 2004.

DUTRA, A. MOLIN, L. H. D. Estudo de caso: Proposta de modelo multicritério de avaliação de desempenho: um estudo de caso na secretaria municipal de indústria e comércio de Tubarão/SC. Revista Interdisciplinar Científica Aplicada, Blumenau,v.7, n.1, p.15-47, 2013.
FRANKLIN, R. B.; FERNANDES, P. P. Métodos de tomada de decisão para empresas de pequeno e médio porte: uma abordagem multicritério. Revista ADMpg Gestão Estratégica, Ponta Grossa, v. 5, n. 1, p.129-136, 2012.

GOMES JÚNIOR, S. F.; CHAVES, M. C. de C.; PEREIRA, E. R.; MELLO, J. C. C. B. S. de; LIMA, G. B. A. Integração de métodos multicritério na busca da sustentabilidade agrícola para a produção de tomates no município de São José de UbáRJ. Pesquisa Operacional, Rio de Janeiro, v. 31, n. 1, p.157$171,2011$.

MORAIS, D. C.; ALMEIDA, A. T. de. Modelo de decisão em grupo para gerenciar perdas de água. Pesquisa. Operacional, Rio de Janeiro, v. 26, n. 3, p. 567-584. 2006.

MORAIS, D. C.; CAVAlCANTE, C. A. V.; ALMEIDA, A. T. de. Priorização de áreas de controle de perdas em redes de distribuição de água. Pesquisa Operacional, Rio de Janeiro, v.30, n.1, p.15-32, 2010.

MOURA, J. A. de; COSTA, A. P. C. S.. Modelo de Decisão Multicritério para Priorização de Projetos de Sustentabilidade. In: XXXII Encontro Nacional de Engenharia de Produção, Desenvolvimento Sustentável e Responsabilidade Social, Bento Gonçalves. Anais ... Bento Gonçalves, 2012. p. 1-9.

POMPERMAYER, R. de S.; PAULA JÚNIOR, D. R. de; NETTO, O.de M. C. Análise Multicritério como Instrumento de Gestão de Recursos Hídricos: O Caso das Bacias dos Rios Piracicaba, Capivari e Jundiaí. Revista Brasileira de Recursos Hídricos, Porto Alegre, v. 12, n. 3, p. 117-127, 2007.

SANTOS, R. B. Avaliação de Intervenções Hidráulicas na Bacia do Rio Gramame-PB com o Uso das Técnicas de Análise Multiobjetivo e Multicriterial. 2009. 202 f. Tese (Doutoradoem Recursos Naturais) - Universidade Federal de Campina Grande, Campina Grande. 2009.

SCIENTEC, Associação para Desenvolvimento da Ciência e Tecnologia. Plano Diretor de Recursos Hídricos da Paraíba: Bacias do Rio Piancó e do Alto Piranhas. SEPLAN. Paraíba. Brasil. 1997.

ZUFFO, A. C.;REIS, L. F. R.; SANTOS R. F.dos; CHAUDHRY, F. H. Aplicação de Métodos Multicritérios ao Planejamento de Recursos Hídricos. Revista Brasileira de Recursos Hídricos, v. 7, n. 1, p.81-102, 2002. 